\title{
Risk factors and clinical features of deterioration in COVID-19 patients in Zhejiang, China: a single-centre, retrospective study
}

Ping $\mathrm{Yi}^{1 \dagger}$, Xiang Yang ${ }^{2+}$, Cheng Ding ${ }^{1}$, Yanfei Chen ${ }^{1}$, Kaijin $\mathrm{Xu}^{1}$, Qing $\mathrm{Ni}^{1}$, Hong Zhao ${ }^{1}$, Yongtao $\mathrm{Li}^{1}$, Xuan Zhang ${ }^{1}$, Jun Liu', Jifang Sheng ${ }^{1}$ and Lanjuan $\mathrm{Li}^{1 *}$

\begin{abstract}
Background: Severe acute respiratory syndrome coronavirus 2 (SARS-CoV-2) infection swept through Wuhan and spread across China and overseas beginning in December 2019. To identify predictors associated with disease progression, we evaluated clinical risk factors for exacerbation of SARS-CoV-2 infection.

Methods: A retrospective analysis was used for PCR-confirmed COVID-19 (coronavirus disease 2019)-diagnosed hospitalized cases between January 19, 2020, and February 19, 2020, in Zhejiang, China. We systematically analysed the clinical characteristics of the patients and predictors of clinical deterioration.

Results: One hundred patients with COVID-19, with a median age of 54 years, were included. Among them, 49 patients (49\%) had severe and critical disease. Age ([36-58] vs [51-70], $P=0.0001)$; sex (49\% vs 77.6\%, $P=0.0031$ ); Body Mass Index (BMI) ([21.53-25.51] vs [23.28-27.01], $P=0.0339)$; hypertension (17.6\% vs $57.1 \%, P<0.0001)$; IL-6 ([6.42-30.46] vs [16.2-81.71], $P=0.0001) ; \mathrm{IL}-10$ ([2.16-5.82] vs [4.35-9.63], $P<0.0001)$; T lymphocyte count ([305$1178]$ vs [167.5-440], $P=0.0001$ ); B lymphocyte count ([91-213] vs [54.5-163.5], $P=0.0001$ ); white blood cell count ([3.9-7.6] vs [5.5-13.6], $P=0.0002)$; D2 dimer ([172-836] vs [408-953], $P=0.005), P C T$ ([0.03-0.07] vs [0.04-0.15], $P=$ $0.0039) ; \operatorname{CRP}([3.8-27.9]$ vs $[17.3-58.9], P<0.0001) ; A S T$ ([16, 29] vs $[18,42], P=0.0484) ;$ artificial liver therapy (2\% vs $16.3 \%, P=0.0148)$; and glucocorticoid therapy $(64.7 \%$ vs $98 \%, P<0.0001)$ were associated with the severity of the disease. Age and weight were independent risk factors for disease severity.

(Continued on next page)
\end{abstract}

\footnotetext{
* Correspondence: ljil@zju.edu.cn

${ }^{+}$Ping Yi and Xiang Yang contributed equally to this work.

'State Key Laboratory for Diagnosis and Treatment of Infectious Diseases, National Clinical Research Centre for Infectious Diseases, Collaborative Innovation Centre for Diagnosis and Treatment of Infectious Diseases, the First Affiliated Hospital, College of Medicine, Zhejiang University, Qingchun Road 79, Hangzhou 31003, Zhejiang Province, People's Republic of China Full list of author information is available at the end of the article
}

(c) The Author(s). 2020 Open Access This article is licensed under a Creative Commons Attribution 4.0 International License, which permits use, sharing, adaptation, distribution and reproduction in any medium or format, as long as you give appropriate credit to the original author(s) and the source, provide a link to the Creative Commons licence, and indicate if changes were made. The images or other third party material in this article are included in the article's Creative Commons. licence, unless indicated otherwise in a credit line to the material. If material is not included in the article's Creative Commons licence and your intended use is not permitted by statutory regulation or exceeds the permitted use, you will need to obtain permission directly from the copyright holder. To view a copy of this licence, visit http://creativecommons.org/licenses/by/4.0/. The Creative Commons Public Domain Dedication waiver (http://creativecommons.org/publicdomain/zero/1.0/) applies to the data made available in this article, unless otherwise stated in a credit line to the data. 
(Continued from previous page)

Conclusion: Deterioration among COVID-19-infected patients occurred rapidly after hospital admission. In our cohort, we found that multiple factors were associated with the severity of COVID19. Early detection and monitoring of these indicators may reduce the progression of the disease. Removing these factors may halt the progression of the disease. In addition, Oxygen support, early treatment with low doses of glucocorticoids and artificial liver therapy, when necessary, may help reduce mortality in critically ill patients.

Keywords: Coronavirus disease 2019 (COVID-19), Severe acute respiratory syndrome coronavirus 2 (SARS-CoV-2), Predictors, Severe illnesses

\section{Background}

In December 2019, a new type of coronavirus disease (COVID-19) occurred in Wuhan, China, which was caused by a novel enveloped RNA betacoronavirus 2 [1]. Due to its phylogenetic similarity to severe acute respiratory syndrome coronavirus (SARS-CoV), COVID-19 was also named SARS-CoV-2 [2]. SARS-CoV-2 infection has become a public health emergency of international concern since it has rapidly spread within China and in several other countries. As of 1 pm 19 February 2020, a total of 74,280 cases of COVID-19 were confirmed in China, with a total of 2008 deaths [3]. The latest mortality was approximately $2.3 \%$ [4]. The infection is highly contagious and can be transmitted from person to person, leading to cluster transmission in families [5]. However, Clinical manifestations of SARS-CoV-2 range from asymptomatic to severe acute respiratory syndrome [1]. Due to these unknown factors, there is currently no specific treatment for SARS-CoV-2, and the risk of death for severely ill patients is very high. Early detection of severe cases can reduce mortality. In this paper, a retrospective analysis of inpatients diagnosed with SARSCoV-2 in Hangzhou, Zhejiang, China, over the period from January 19, 2020, to February 19, 2020, was performed to explore the relevant factors that may predict the risk factors for disease severity.

\section{Method}

\section{Study design and participants}

We included in the study all patients diagnosed with SARS-CoV-2 who were admitted to the First Affiliated Hospital of Zhejiang University School of Medicine between January 19, 2020, and February 19, 2020. This study was approved by the ethics review committee of the First Affiliated Hospital of Zhejiang University and conforms to the code of ethics of the Helsinki declaration in 2013.

The degree of severity of SARS-CoV-2 (severe vs nonsevere) at admission was assessed using the latest guidelines for SARS-CoV-2 diagnosis from the National Health Commission of China [6]. As outlined in the guidelines, patients were classified into four types: mild, moderate, severe, and critical illness. Mild cases included patients with mild clinical symptoms and no signs of pneumonia on imaging. Moderate cases included patients with fever, respiratory symptoms, and imaging manifestations of pneumonia. Severe cases included patients who met one of the following criteria: respiratory rate $\geq 30$ breaths per minute; arterial oxygen saturation $(\mathrm{SaO} 2) \leqq 93 \%$ at rest; or partial pressure of oxygen $(\mathrm{PaO} 2) /$ Fraction of Inspiration O2 (FiO2) $\leqq 300 \mathrm{mmHg}$. Critical illness cases included patients who met any of the following criteria: respiratory failure requiring mechanical ventilation, occurrence of shock, and complications with other organ failure requiring care in an intensive care unit. Mild and moderate cases were categorized as non-severe cases, while severe and critical illness cases were defined as severe cases in this study.

\section{Cytokine and lymphocyte measurement}

Plasma cytokines (IL6, IL10) were measured in all patients using the Human Cytokine standard 27-plex assay panel and the Bio-PLEX 200 system (Bio-RAD, Hercules, CA, USA) according to the manufacturer's instructions. Lymphocyte subset counts in peripheral blood [CD45+, CD3+ CD4+, CD3-CD19+ and CD3-CD16+ CD56+ ]were measured by flow cytometry in accordance with the manufacturer's protocol. (BD FACS Calibur, USA). They were completed by the laboratory department of our hospital and the State Key Laboratory of Diagnosis and Treatment of infectious diseases.

\section{Data collection}

Data were collected from electronic medical records for the following parameters: demographics, underlying medical comorbidities, clinical symptoms and signs, laboratory tests, treatment. Laboratory tests consisted of IL-6, IL-10, lymphocyte subset count, white blood cell count, D2 dimer, PCT, CRP, and AST. Underlying medical comorbidities mainly included hypertension, diabetes and coronary heart disease. Treatment included antiviral, ambroxol, glucocorticoid, and artificial liver therapy.

\section{SARS-CoV-2 RT-PCR test}

Respiratory tract samples, such as nasopharyngeal, sputum, or throat swab samples, from all included patients 
were collected daily (Nasopharyngeal or throat swab sampling is less than $10 \%$ of all samples). Using SARSCoV-2 nucleic acid detection kits (Shanghai Biogerm Medical Technology Co Ltd), we detected SARS-CoV-2 viral RNA by polymerase chain reaction analysis in accordance with the manufacturer's protocol. The diagnosis was confirmed by the criteria recommended by the National Institute for Viral Disease Control and Prevention (China). A viral cycle threshold (CT) value less than 37 was defined as positive, and a CT value greater than 40 was defined as negative. A CT value between 37 and 40 was confirmed by retesting.

\section{Statistics analyses}

Continuous variables are presented as the median (Interquartile range IQR) and were compared by the KruskalWallis test between the non-severe group and the severe group. Categorical variables were presented as numbers (\%) and compared by the chi-square $\left(x^{2}\right)$ test or Fisher's exact test. The Kaplan-Meier (product-limit) method was used to evaluate the time periods from glucocorticoid treatment and artificial liver therapy to different clinical outcomes between the two groups. All statistical analyses were performed with SAS 9.4 software (SAS Institute Inc., Cary, NC, USA). The significance level of the hypotheses tests was set at 0.05 (two-sided).

\section{Patient and public participation}

This was a retrospective cohort study in which the patients were not involved in the design of the study, the setting of the study questions, or the direct measurement of the results.

\section{Result}

\section{Patient characteristics on admission}

Of the 100 hospitalized SARS-CoV-2 patients between January 19, 2020, and February 19, 2020, 49 (49\%) patients were critically ill. The demographic and clinical characteristics are shown in Table 1 . The median age was 54 years. The age (median) of the severe group was significantly higher than that of the non-severe group (61 [51-70] vs 50 [36-58]; $P=0.0001])$. Sixty-three percent of the patients were older than 50 years of age. A total of $63 \%$ of patients were male. In the severe group, the proportion of males was significantly higher than that of females $(77.6 \%$ vs $49 \% ; P=0.0031)$. Overweight (BMI > 24) patients were common in both groups of COVID-19 patients. BMI was significantly higher in the severely ill group (24.44 (23.28-27.01) vs 24 (21.5325.51); $P=0.0339$ ). Underlying medical conditions such as hypertension (37\%) and diabetes mellitus (11\%) were the most common coexisting illnesses among SARSCoV-2 patients. Moreover, the presence of hypertension was more common among patients with severe disease
(57.1\% vs $17.6 ; P<0.0001)$. Fever was present in $71 \%$ of our patients, and there was no significant difference in fever between the two groups. In our study, the vast majority of patients (98\%) had obvious pulmonary CT lesions. Especially in severe patients, pulmonary computed tomography (CT) lesions of different degrees were observed on admission, including patchy, nodular ground glass shadows and interstitial abnormalities.

\section{Laboratory tests}

Among laboratory indicators at admission, the white blood cell count ([3.9-7.6] vs [5.5-13.6], $P=0.0002)$, Ddimer ([172-836] vs [408-953], $P=0.005)$, C-reactive protein $([3.8-27.9]$ vs $[17.3-58.9], P<0.0001)$, procalcitonin $([0.03-0.07]$ vs $[0.04-0.15], P=0.0039)$ and alanine aminotransferase $([16,29]$ vs $[18,42], P<0.0484)$ in the non-severe group were significantly lower than those in the severe group. In our study, we mainly focused on the laboratory detection of cytokines and immune cell subsets in patients with SARS-CoV-2 infection. We found that the expression of IL- 6 in severe patients was significantly higher than that in the non-severe group ([6.42$30.46]$ vs [16.2-81.71], $P=0.0001)$, while the expression of IL-10 in severe patients was significantly lower than that in the non-severe group ([2.16-5.82] vs [4.35-9.63], $P<0.0001)$. The total number of T cells $([305-1178]$ vs [167.5-440], $P<0.0001), B$ cells $([91-213]$ vs [54.5163.5], $P=0.0189)$, absolute number of lymphocytes ([620-1554] vs [367.5-810.5], $P=0.0002)$, Th ([176618] vs [60.5-214.5], $P<0.0001)$ and Ts/Tc cells ([137443)] vs [69-140.5], $P<0.0001)$ were significantly lower than those in non-severe cases.

\section{Treatment and clinical outcomes}

The treatment and clinical outcomes of COVID-19 patients are shown in Table 2. All 100 patients received antiviral treatment including lopinavir/ritonavir, interferon- $\alpha$, darunavir/cobicistat, favipiravir and arbidol. The median time from symptom onset to antiviral regimen administration was 7 (4-9.5) days, with no significant difference between the non-severe and severe groups. The median time from antiviral regimen administration to negative results for SARS-CoV-2 RNA for the first time was $9(5-14)$ days. Moreover, the time in the non-severe group was significantly shorter than that in the severe group $(6(4-12)$ vs $9(7-15)$ days; $P=$ $0.0142)$. The median duration from antiviral treatment to the time of a negative viral testing result was 10 (615) days, and the duration of the non-severe group was significantly shorter than that of the severe group (7 (413) vs $12(9-18) ; P=0.0006)$. In addition, our patients received supportive symptomatic treatments, including oxygen, glucocorticoid, ambroxol, antibiotic, and artificial liver therapy. Glucocorticoids were given to 80 
Table 1 Clinical Characteristics of Patients With SARS-CoV-2 Infection

\begin{tabular}{|c|c|c|c|c|}
\hline Characteristic & Total $(N=100)$ & Non-Severity $(n=51)$ & Severity $(n=49)$ & $P$ Value* \\
\hline \multicolumn{5}{|l|}{ Demographics, n (\%) } \\
\hline Age, median (IQR), y & $54(42-64)$ & $50(36-58)$ & $61(51-70)$ & 0.0001 \\
\hline$\geq 50$ & $63(63)$ & $26(51)$ & $37(75.5)$ & 0.0111 \\
\hline Male sex & $63(63)$ & $25(49)$ & $38(77.6)$ & 0.0031 \\
\hline BMI & $24.27(22.14-25.97)$ & $24.01(21.53-25.51)$ & $24.44(23.28-27.01)$ & 0.0339 \\
\hline \multicolumn{5}{|l|}{ Mainly underlying conditions, n (\%) } \\
\hline Hypertension & $37(37)$ & $9(17.6)$ & $28(57.1)$ & $<0.0001$ \\
\hline Diabetes mellitus & $11(11)$ & $3(5.9)$ & $8(16.3)$ & 0.3574 \\
\hline Cardiac disease & $4(4)$ & $1(2)$ & $3(6)$ & 0.118 \\
\hline \multicolumn{5}{|l|}{ Fever on admission- ${ }^{\circ} \mathrm{C}$} \\
\hline$<37.5^{\circ} \mathrm{C}$ & $29(29)$ & 19 (37.3) & $10(20.4)$ & 0.0635 \\
\hline \multicolumn{5}{|l|}{ Fever during hospitalization } \\
\hline Median highest temperature (IQR) & $38.25(37.5-39)$ & $38.2(37.6-39)$ & $38.3(37.5-39)$ & 0.9669 \\
\hline \multicolumn{5}{|l|}{ Radiologic findings on admission } \\
\hline \multicolumn{5}{|l|}{ chest CT — no./total no. (\%) } \\
\hline Normal & $2(2)$ & $2(3.9)$ & 0 & 0.4952 \\
\hline \multicolumn{5}{|l|}{ Initial laboratory findings, median (IQR) } \\
\hline WBC, $10^{9} / \mathrm{L}$ & $6.4(4.1-10.8)$ & $5.15(3.9-7.6)$ & $9.1(5.5-13.6)$ & 0.0002 \\
\hline d-dimer & $452.5(240-857.5)$ & $339(172-836)$ & $604(408-953)$ & 0.005 \\
\hline ALT & $22(15-40.5)$ & $20(13-41)$ & $24(15-40)$ & 0.2801 \\
\hline AST & $20.5(16-34)$ & $20(16-29)$ & $25(18-42)$ & 0.0484 \\
\hline CRP & $24.665(7.785-47.6)$ & $10.9(3.8-27.9)$ & $39.78(17.3-58.9)$ & 0.0001 \\
\hline PCT & $0.06(0.03-0.1)$ & $0.04(0.03-0.07)$ & $0.07(0.04-0.15)$ & 0.0039 \\
\hline IL2 & $0.95(0.855-1.82)$ & $0.95(0.78-1.83)$ & $0.95(0.95-1.81)$ & 0.5534 \\
\hline IL6 & $20.425(8.78-56.04)$ & $12.52(6.42-30.46)$ & $38.22(16.2-81.71)$ & 0.0001 \\
\hline IL10 & $4.775(2.91-8.03)$ & $3.45(2.16-5.82)$ & $6.93(4.35-9.63)$ & $<0.0001$ \\
\hline TNF-a & $17.98(10.945-59.085)$ & $23.29(12.2-65.49)$ & $12.22(10.77-36.44)$ & 0.1658 \\
\hline IFN-r & $10.06(5.12-33.56)$ & $11.4(4.92-39.71)$ & $9.82(5.19-28.57)$ & 0.6943 \\
\hline $\mathrm{ALC}$ & 703 (402-1119) & 1119 (620-1554) & $543.5(367.5-810.5)$ & 0.0002 \\
\hline Total T cells & $348(233-775)$ & 752 (305-1178) & $276.5(167.5-440)$ & 0.0001 \\
\hline Th cells & $176(79-431)$ & $376(176-618)$ & $115(60.5-214.5)$ & $<0.0001$ \\
\hline B cells & $115(70-211)$ & $167(91-213)$ & $93.5(54.5-163.5)$ & 0.0189 \\
\hline NK cells & 105 (64-183) & 154 (70-207) & $101(61.5-155.5)$ & 0.1201 \\
\hline
\end{tabular}

Abbreviation: IQR Inter quartile range, BMI Body mass index, WBC White blood count, ALT Aspartate aminotransferase, AST Alanine aminotransferase, CRP C-reactive protein, PCT Procalcitonin, IL Interleukin, TNF-a Tumor necrosis factor a, IFN Interferon, Th T helper cells, ALC Absolute lymphocyte count, NK cells Natural killer cells *, Chi-square $\left(X^{2}\right)$ test or Fisher's exact test was used with $P<0.05$ as significant

(80\%) patients, and the proportion was significantly higher in the severe group than in the non-severe group (98\% vs 64.7\%; $P<0.0001$ ). Meanwhile, the maximum dosage of glucocorticoids in the severe group was significantly higher than that in the non-severe group (40[40-80] vs 40 [0-40]; $P=0.0001)$. Compared with the non-severe group, the severe group had a higher rate of antibiotic treatment $(44.9 \%$ vs $3.9 \% ; P<.0001)$. In the retrospective analysis, we found that the clinical outcome of our patients was very good. No patients died, and $86 \%$ of the patients recovered and were discharged from the hospital. The discharge rate of the non-severe group was significantly higher than that of the severe group (96.1\% vs $75.5 \% ; P=0.003)$. The Intensive Care Unit (ICU) admission rate was $23 \%$, which was higher in the severe group (42.9\% vs $3.9 \% ; P<0.0001)$.

\section{Risk factors for SARS-CoV-2 severe illness}

Using a multivariate logistic regression analysis, we identified the risk factors associated with exacerbation of 
Table 2 Comparison of treatment responses and clinical outcomes of patients infected with SARS-CoV-2 between non-severe and severe group

\begin{tabular}{|c|c|c|c|c|}
\hline Variable & Total $(N=100)$ & Non-Severity $(n=51)$ & Severity $(n=49)$ & $P$ Value \\
\hline \multicolumn{5}{|l|}{ Treatments $\mathrm{n}(\%)$} \\
\hline Antivirus treatment & $100(100)$ & $51(100)$ & $49(100)$ & \\
\hline \multicolumn{5}{|l|}{ median (IQR), days } \\
\hline Time from illness onset to Antivirus start & $7(4-9.5)$ & $6(3-9)$ & $7(5-10)$ & 0.2948 \\
\hline Glucocorticoid treatment n (\%) & $81(81)$ & $33(64.7)$ & $48(98)$ & $<0.0001$ \\
\hline Maximum dosage & $80(20-80)$ & $80(0-40)$ & $80(40-80)$ & 0.0001 \\
\hline \multicolumn{5}{|c|}{ (equivalent methylprednisolone), median (IQR), mg/d } \\
\hline Artificial liver support n (\%) & $9(9)$ & $1(2)$ & $8(16.3)$ & 0.0148 \\
\hline Antibiotic treatment $\mathrm{n}(\%)$ & $24(24)$ & $2(3.9)$ & $22(44.9)$ & $<0.0001$ \\
\hline Oxygen support & & & & $<0.0001$ \\
\hline Nasal cannular & $64(64)$ & $46(90.2)$ & $18(36.7)$ & \\
\hline high-flow nasal cannula & $13(13)$ & $3(5.9)$ & $10(20.4)$ & \\
\hline Invasive mechanical ventilation & $23(23)$ & $2(3.9)$ & $21(42.9)$ & \\
\hline \multicolumn{5}{|l|}{ median (IQR), days } \\
\hline${ }^{\mathrm{A}} \mathrm{AT}$ to first virologic conversion & $9(5-14)$ & $6(4-12)$ & $9(7-15)$ & 0.0142 \\
\hline AT to stable virologic conversion & $10(6-15)$ & $7(4-13)$ & $12(9-18)$ & 0.0006 \\
\hline AT to radiologic recovery & $7(4-10)$ & $7(5-11)$ & $6.5(4-9.5)$ & 0.3162 \\
\hline AT to temperature recovery & $5(2-8)$ & $3(2-7)$ & $6.5(2-9)$ & 0.0903 \\
\hline \multicolumn{5}{|l|}{ Clinical outcomes, n (\%) } \\
\hline Discharge from hospital & $86(86)$ & $49(96.1)$ & $37(75.5)$ & 0.0030 \\
\hline${ }^{B} \mathrm{ICU}$ admission & $23(23)$ & $2(3.9)$ & $21(42.9)$ & $<0.0001$ \\
\hline Death & 0 & 0 & 0 & 0 \\
\hline
\end{tabular}

Abbreviation: ${ }^{\mathrm{A}} \mathrm{A} T$ Antiviral therapy onset, ${ }^{\mathrm{B}} \mathrm{C} U$ Intensive care unit

SARS-CoV-2 (Table 3). Age and BMI were recognized as predictors (independent factors) of severe illness. However, sex, hypertension, IL-6, T lymphocyte count, B lymphocyte count, glucocorticoid treatment and artificial liver support were not recognized as independent factors.

\section{Discussion}

In December 2019, a new infectious disease, SARS-CoV2 , swept through Wuhan and quickly spread to all Chinese cities and dozens of countries overseas. Similar diseases are highly infectious and can spread through human-to-human transmission among close contacts and over a wide area [7, 8]. However, the source of SARS-CoV-2 is not fully clear, and the lack of specific treatments may cause the patient's symptoms to progress from mild to severe or even result in death. Previous studies reported that the fatality rate among hospitalized SARS-CoV-2 patients was approximately $2.3 \%$, which was much lower than those of SARS- and Middle East Respiratory Syndrome (MERS)-infected patients $[4,9]$. For such patients, we need to remain vigilant and provide symptomatic supportive treatment early to reduce the occurrence of severe disease.

In our retrospective cohort study, we included 100 SARS-CoV-2 patients from a single clinical centre in

Table 3 Multivariate Logistic Regression analysis of risk factor for disease severity among hospitalized patients with COVID19

\begin{tabular}{lll}
\hline Variable & Odds Ratio $(\mathbf{9 5 \%}$ Confidence Interval) & $\boldsymbol{P}$ Value \\
\hline Age & $1.064(1.007-1.124)$ & $\mathbf{. 0 2 7}$ \\
BMI & $1.240(1.006-1.528)$ & $\mathbf{. 0 4 4}$ \\
IL6 & $1.005(0.995-1.015)$ & .307 \\
T cells & $1.003(0.995-1.011)$ & .424 \\
B cells & $1.005(0.997-1.014)$ & .196 \\
Artificial liver support & $0.985(0.073-13.211)$ & .99 \\
\hline
\end{tabular}


Hangzhou, Zhejiang Province. Our results showed that age and BMI were independent risk factors for severe illness. The median patient age was 54 years. Sixty-three percent of the patients were older than 50 years, which was consistent with multiple reports in the literature $[10,11]$. Severe patients were much older than nonsevere patients, which may be related to a lower immune response and a higher frequency of underlying conditions that are not beneficial for self-limited recovery after virus infection. In addition, we discovered that overweight (BMI > 24) was an important risk factor for severity in SARS-CoV-2 patients. Compared with the BMI of severe patients, non-severe patients had a lower $\mathrm{BMI}$, with significant differences between the two groups $(P=0.0339)$.

Similar to H7N9, the most common symptoms of SARS-CoV-2 patients were fever and cough [5, 12]. However, $26 \%$ of SARS-CoV-2 patients who were admitted to our hospital had a normal temperature. If these patients had been overlooked as having SARS-CoV-2, then more people might have been infected. This was similar to previous reports [13]. Coexisting disorders such as hypertension and diabetes mellitus were associated with severe illnesses. The proportions of hypertension and diabetes mellitus in severe illnesses were higher than those in non-severe cases. Furthermore, hypertension was significantly different between the two groups $(P<0.0001)$. This was consistent with H7N9 patients [14]. In addition, we also found that the laboratory parameters and supplementary treatment, including the white blood cell count, D-dimer, C-reactive protein, procalcitonin, alanine aminotransferase, IL-6, IL-10, CD3+ $T$-cell, CD4+ T-cell $(\mathrm{CD} 3+\mathrm{CD} 4+)$, CD8+ $T$-cell $(\mathrm{CD} 3+$ CD8+), B-cell (CD3-CD19+) and NK-cell (CD3CD16 + CD56+), absolute lymphocyte number, and TS/ Tc cells and the need for antibiotic, glucocorticoid and artificial liver therapy, were identified in single variable analysis as risk factors for severe illnesses but that these risk factors did not reach our criteria for significance in the multivariate analysis.

Previous studies found that an inflammatory factor storm was one of the important factors for severe illness and even death of H7N9, SARS-CoV, MERS-CoV. The mechanism may be related to the overexpression of inflammatory factors and chemokines, which can lead to acute lung injury and ARDS $[1,15,16]$. In our retrospective study, we also found a significant increase in cytokine IL-6 in the patients with severe disease. Therefore, for patients with a gradual increase in the expression of such inflammatory factors, early low-dose glucocorticoid and artificial liver treatment may alleviate the progression of the disease and reduce the risk of death. The effectiveness and necessity of glucocorticoid use has been controversial in novel coronavirus infections. Large doses of glucocorticoids may cause significant side effects such as lymphocytopenia and femoral head necrosis [17]. In our cohort, the rates of treatments with glucocorticoids and artificial liver therapy in the severe group were greater than those in the non-severe group, and there were significant differences. However, there were no statistically significant differences in the treatment effects between the two groups (the days from treatment initiation to virus clearance) (supplementary figures 1-2), which may be related to our small sample size. Further research on larger cohorts is in progress. Our study found that the number of immune cells in most patients with mild disease is normal. The decrease in $\mathrm{T}$ lymphocytes, B lymphocytes and absolute lymphocyte counts are correlated with the severity of the disease. Therefore, early detection of related immune indicators may provide us with the ability to predict the severity of COVID19.

There are several limitations to be considered when interpreting the findings. First, our study is a singlecentre study of SARS-CoV-2 risk factors for critically ill patients in a hospital. Furthermore, when screening confirmed cases, the vast majority of tested samples were from lower respiratory tract specimens, but there were still a few pharyngeal swab specimens, and the pharyngeal swab false negative rate may have led to the lack of included patients. Finally, the number of children under 18 years in our sample was small, so no specific conclusions could be drawn for adolescents.

\section{Conclusions}

Patients with SARS-CoV-2 infection are likely to progress to critical illness. In our analysis, we found that pre-existing diseases and multiple laboratory indicators were associated with disease progression. Age and BMI may be independent risk factors for the development of severe SARS-CoV-2. Therefore, it is important to evaluate the severity of the newly diagnosed patients' condition to provide individualized diagnosis and treatment and to improve the prognosis.

\section{Supplementary Information}

The online version contains supplementary material available at https://doi. org/10.1186/s12879-020-05682-4.

\section{Additional file 1.}

\section{Abbreviations}

COVID-19: Coronavirus Disease 2019; SARS-CoV-2: Severe Acute Respiratory Syndrome Coronavirus 2; SaO2: Arterial Oxygen Saturation; PaO2: Partial

Pressure of Oxygen; FiO2: Fraction of Inspiration O2; CT value: Cycle Threshold Value; IQR: Interquartile range; BMI: Body Mass Index; CT: Computed Tomography; ICU: Intensive Care Unit 


\section{Acknowledgments}

The authors thank the staff at the hospitals in Zhejiang, provinces for providing assistance with field investigation administration and data collection.

\section{Authors' contributions}

PY and $X Y$ contributed equally to this article. $K J X$ and $L J L$ conceptualized the paper. CD analysed the data, with input from JFS, KJX, YFC, CD, QN, YTL, XZ, JL.PY and XY wrote the initial draft with all authors providing critical feedback and edits to subsequent revisions. All authors approved the final draft of the manuscript. L- $L$ is the guarantor. The corresponding author attests that all listed authors meet authorship criteria and that no others meeting the criteria have been omitted.

\section{Funding}

This work was supported by Natural Science Foundation of Zhejiang Province (LY20H030008), Science and Technology Department Key Research and Development Plan Emergency Project of Zhejiang Province (2020c03123-1), Natural Science and Technology Major Project (2017ZX1024401001002). And the funding source did not interfere with the design of the study and collection, analysis, and interpretation of data and in writing the manuscript.

\section{Availability of data and materials}

The datasets used and/or analyzed during the current study are available from the corresponding author and data was publicly available on reasonable request.

\section{Ethics approval and consent to participate}

This study was approved by the ethics review committee of the First Affiliated Hospital of Zhejiang University and conforms to the code of ethics of the Helsinki declaration in 2013. Written informed consent was obtained from the patients.

\section{Consent for publication}

Not Applicable.

\section{Competing interests}

The authors declare that they have no competing interests.

\section{Author details}

State Key Laboratory for Diagnosis and Treatment of Infectious Diseases, National Clinical Research Centre for Infectious Diseases, Collaborative Innovation Centre for Diagnosis and Treatment of Infectious Diseases, the First Affiliated Hospital, College of Medicine, Zhejiang University, Qingchun Road 79, Hangzhou 31003, Zhejiang Province, People's Republic of China. ${ }^{2}$ Department of Infectious Disease, ShuLan Hangzhou Hospital, Hangzhou, Zhejiang Province, China.

Received: 10 April 2020 Accepted: 3 December 2020

Published online: 10 December 2020

\section{References}

1. Huang C, Wang Y, Li X, Ren L, Zhao J, Hu Y, Zhang L, Fan G, Xu J, Gu X, et al. Clinical features of patients infected with 2019 novel coronavirus in Wuhan, China. Lancet. 2020;395:497-506.

2. Zhu N, Zhang D, Wang W, Li X, Yang B, Song J, Zhao X, Huang B, Shi W, Lu $\mathrm{R}$, et al. A novel coronavirus from patients with pneumonia in China, 2019. N Engl J Med. 2020;382(8):727-33.

3. WHO 2019 nCoV situation report. Available from:https:/www.who.int/docs/ default-source/coronaviruse/situation-reports/20200204-sitrep-15-ncov. pdf?sfvrsn=88fe8ad6_2.

4. Wu Z, McGoogan JM. Characteristics of and important lessons from the coronavirus disease 2019 (COVID-19) outbreak in China: summary of a report of 72314 cases from the Chinese Center for Disease Control and Prevention. JAMA. 2020;323:1239-42.

5. Guan WJ, Ni ZY, Hu Y, Liang WH, Ou CQ, He JX, Liu L, Shan H, Lei CL, Hui DSC, et al. Clinical characteristics of coronavirus disease 2019 in China. N Engl J Med. 2020;382:1708-20.

6. Clinical management of 2019 nCoV guidelines by National Health Commission of the People Republic of China. Available from: [http:// www.nhc.gov.cn/yzygj/s7652m/202003/a31191442e29474b98bfed5579d5 af95.shtml].

7. Li Q, Guan X, Wu P, Wang X, Zhou L, Tong Y, Ren R, Leung KSM, Lau EHY, Wong JY, et al. Early transmission dynamics in Wuhan, China, of novel coronavirus-infected pneumonia. N Engl J Med. 2020;382:1199-207.

8. Chen N, Zhou M, Dong X, Qu J, Gong F, Han Y, Qiu Y, Wang J, Liu Y, Wei Y, et al. Epidemiological and clinical characteristics of 99 cases of 2019 novel coronavirus pneumonia in Wuhan, China: a descriptive study. Lancet. 2020; 395(10223):507-13.

9. Deng SQ, Peng HJ. Characteristics of and Public Health Responses to the Coronavirus Disease 2019 Outbreak in China. J Clin Med. 2020;9(2):575.

10. Zhang JJ, Dong X, Cao YY, Yuan YD, Yang YB, Yan YQ, Akdis CA, Gao YD. Clinical characteristics of 140 patients infected with SARS-CoV-2 in Wuhan, China. Allergy. 2020;75:1730.

11. Jiang F, Deng L, Zhang L, Cai Y, Cheung CW, Xia Z. Review of the clinical characteristics of coronavirus disease 2019 (COVID-19). J Gen Intern Med. 2020;35(5):1545-9.

12. Gao HN, Lu HZ, Cao B, Du B, Shang H, Gan JH, Lu SH, Yang YD, Fang Q, Shen $Y Z$, et al. Clinical findings in 111 cases of influenza a (H7N9) virus infection. N Engl J Med. 2013;368(24):2277-85.

13. Hu Z, Song C, Xu C, Jin G, Chen Y, Xu X, Ma H, Chen W, Lin Y, Zheng Y, et al. Clinical characteristics of 24 asymptomatic infections with COVID-19 screened among close contacts in Nanjing, China. Sci China Life Sci. 2020; 63:706-11.

14. Wang C, Yu H, Horby PW, Cao B, Wu P, Yang S, Gao H, Li H, Tsang TK, Liao $\mathrm{Q}$, et al. Comparison of patients hospitalized with influenza a subtypes H7N9, H5N1, and 2009 pandemic H1N1. Clin Infect Dis. 2014;58(8):1095-103.

15. Channappanavar R, Perlman S. Pathogenic human coronavirus infections: causes and consequences of cytokine storm and immunopathology. Semin Immunopathol. 2017;39(5):529-39.

16. Yu M, Wang Q, Qi W, Zhang K, Liu J, Tao P, Ge S, Liao M, Ning Z. Expression of inflammation-related genes in the lung of BALB/C mice response to H7N9 influenza a virus with different pathogenicity. Med Microbiol Immunol. 2016;205(5):501-9.

17. Panesar NS. What caused lymphopenia in SARS and how reliable is the lymphokine status in glucocorticoid-treated patients? Med Hypotheses. 2008;71(2):298-301.

\section{Publisher's Note}

Springer Nature remains neutral with regard to jurisdictional claims in published maps and institutional affiliations.

\section{Ready to submit your research? Choose BMC and benefit from}

- fast, convenient online submission

- thorough peer review by experienced researchers in your field

- rapid publication on acceptance

- support for research data, including large and complex data types

- gold Open Access which fosters wider collaboration and increased citations

- maximum visibility for your research: over $100 \mathrm{M}$ website views per year

At BMC, research is always in progress.

Learn more biomedcentral.com/submissions 